\title{
Transient growth analysis of the flow past a circular cylinder
}

\author{
N. Abdessemed, A. S. Sharma, S. J. Sherwin, and V. Theofilis \\ Department of Aeronautics, Imperial College London. South Kensington. \\ London SW7 2AZ, United Kingdom \\ Departntent of Electrical and Electronic Engineering. Inperial College London, \\ South Kensington. London SW7 2AZ. Uniteal Kingdom \\ School of Aeronautics, Universidad Politécnica de Madrid, Pza. Cardenal Cisneros 3. \\ E28040 Madrid. Spain
}

\begin{abstract}
We apply direct transient growth analysis in complex geometries to investigate its role in the primary and secondary bifurcation/transition process of the flow past a circular cylinder. The methodology is based on the singular value decomposition of the Navier-Stokes evolution operator linearized about a two-dimensional steady or periodic state which leads to the optimal growth modes. Linearly stable and unstable steady flow at $\mathrm{Re}=45$ and 50 is considered first, where the analysis demonstrates that strong two-dimensional transient growth is observed with energy amplifications of order of $10^{3}$ at $U_{c s} \tau / D \approx 30$. Transient growth at $\operatorname{Re}=50$ promotes the linear instability which ultimately saturates into the well known von-Kármán street. Subsequently we consider the transient growth upon the time-periodic base state conresponding to the von-Karman street at $\operatorname{Re}=200$ and 300. Depending upon the spanwise wavenumber the flow at these Reynolds numbers are linearly unstable due to the so-called mode $A$ and $B$ instabilities. Once again energy amplifications of order of $10^{3}$ are observed over a time interval of $\tau / T=2$, where $T$ is the time period of the base flow shedding. In all cases the maximum energy of the optimal initial conditions are located within a diameter of the cylinder in contrast to the spatial distribution of the unstable eigenmodes which extend far into the downstream wake. It is therefore reasonable to consider the analysis as presenting an accelerator to the existing modal mechanism. The rapid amplification of the optimal growth modes highlights their importance in the transition process for flow past circular cylinder, particularly when comparing with experimental results where these types of convective instability mechanisms are likely to be activated. The spatial localization, close to the cylinder, of the optimal initial condition may be significant when considering strategies to promote or control shedding.
\end{abstract}

\section{INTRODUCTION}

Classically, the study of hydrodynamic stability has proceeded via linearization and subsequent eigenmodal analysis. ${ }^{1}$ In this approach, the asymptotic behavior of small perturbations to a steady or time-periodic base flow is considered. This asymptotic behavior is necessarily determined by the eigenvalues of a linear operator arising from the analysis, which describes the time evolution of the eigenmodes.

Many canonical problems, such as flow in a channel, permit stability analysis to be performed about a velocity field which depends on a single coordinate. However in more complex geometries one extension to the classical hydrodynamic stability analysis is to use fully resolved computational stability analysis of the flow field. ${ }^{2.3}$ This has been referred to as direct linear stability analysis in analogy to the usage direct numerical simulation (DNS), it has also previously been referred to as biglobal stability analysis. ${ }^{4}$ In this approach one has the ability to fully resolve in two or even three dimensions the base flow, e.g., $\mathbf{U}(x, y, z)$, and to perform a stability analysis with respect to perturbations in two or three dimensions, e.g., $\mathbf{u}^{\prime}=\mathbf{u}^{\prime}(x, y, z, t)$. Typically in this methodology one does not need to resort to any approxima- tions beyond the initial linearization other than perhaps certain inflow and outflow conditions.

In particular one can consider cases with rapid streamwise variation of the flow in two spatial dimensions such as flow past a circular cylinder. By postulating modal instabilities of the form: $\mathbf{u}^{\prime}(x, y, z, t)=\tilde{\mathbf{u}}(x, y) \exp (i \beta z+\lambda t)$ (assuming one direction of homogeneity in the $z$-direction), asymptotic instability analysis becomes a large-scale eigenvalue problem for the modal shape $\overline{\mathbf{u}}$ and eigenvalue $\lambda$. There are algorithms and numerical techniques which allow one to obtain leading (critical or near critical) eigenvalues and eigenmodes for the resulting large problems. ${ }^{3}$ This approach has been found to be extremely effective at determining absolute instabilities in many complex geometry flows, both open and closed $\mathrm{d}^{2,5-13}$ including weakly nonlinear stability. ${ }^{3,14}$

Direct linear stability analysis has not been routinely applied to convective instabilities that commonly arise in open domain problems with inflow and outflow conditions. One reason is that such instabilities are not typically dominated by eigenmodal behavior, but rather by linear transient growth that can arise owing to the non-normality of the eigenmodes. A large-scale eigenvalue analysis simply camot detect such behavior although for streamwise-periodic flow, it is possible 
to analyze convective instability through direct linear stability analysis, see, e.g., Schatz et al. ${ }^{15}$

A more recent extension of hydrodynamic stability analysis is nonmodal stability analysis or transient growth analysis. ${ }^{16-19}$ Here one examines the linear growth of infinitesimal perturbations over a prescribed finite time interval and does not assume eigenmodal growth of the linearized operator. Much of the initial focus in this area has been on large linear transient amplification and the relationship of this to subcritical transition to turbulence in plane shear flows. ${ }^{20.16}$ While this relationship has been previously considered in the context of the Ginzberg-Landau equation, ${ }^{21.22}$ it has not been widely exploited in the type of large-scale direct linear stability analyses that have been successful in promoting the understanding of absolute instabilities in complex flows. This approach has been recently employed in backward-facing-step flows ${ }^{23,24}$ and flows in a constructed (stenotic) pipe. ${ }^{25}$ Also, with different emphasis from the present approach, Ehrenstein and Gallaire ${ }^{26}$ directly computed modes in boundary layer flow to analyze transient growth associated with convective instability and Hrepffner et $a{ }^{27}$ investigated the transient growth of boundary layer streaks. Hill ${ }^{28}$ and Giannetti and Luchini ${ }^{29}$ considered the role of the adjoint operator in steady flow past a cylinder. It is also possible to pose a temporally fixed, spatial varying quadratic transient growth problem, but this approach has not been followed in this work.

Methods developed for direct linear stability analysis of the Navier-Stokes equations in general geometries have been previously described in detail, see Ref. 3 and extensively applied. ${ }^{2,30,10,7,31,12,32}$ Only recently, however, have largescale techniques been extended to the transient growth problem. In Ref. 33 a method for direct optimal growth computation for the linearized Navier-Stokes equations in general geometries was presented. This techniques have been applied to steady flows past a low pressure turbine blades, ${ }^{34}$ a backward-facing step, ${ }^{24}$ and a stenotic/constricted pipe flows. ${ }^{25}$

Barkley and Henderson ${ }^{2}$ applied direct stability analysis to investigate the onset of three-dimensional (3D) secondary instability about the periodic von-Kármán shedding of flow past a circular cylinder. In this work Barkley \& Henderson identified linear Floquet modes associated to long- as well as short-wavelength perturbations referred to as modes $A$ and $B$, respectively. These instabilities were previously observed experimentally by Williamson. ${ }^{35}$ Subsequently a quasiperiodic Floquet mode has also been identified by Blackbum and Lopez. $^{36}$

Although, the Floquet multipliers of modes $A$ and $B$ are characterized by exponential growth, depending on the growth rate, there will necessarily be an initial time interval over which this growth is relatively small. For instance, at $\operatorname{Re}=200$ the maximum instability has a growth rate over one shedding period of $\mu \approx 1.12$, and so it takes approximately 10 shedding cycles for the energy of mode $A$ to grow one order of magnitude. We can therefore ask what mechanisms are likely to dominate during this time interval. In this paper we will therefore consider the relative role of transient growth mechanisms, identified using direct optimal growth (a)
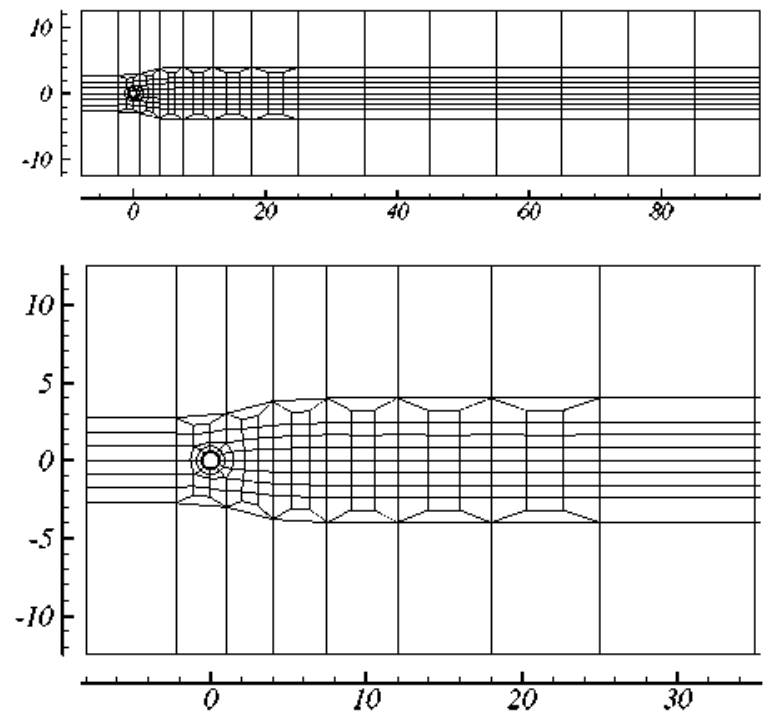

(b)

FIG. 1. Macro-spectral/hp element mesh. (a) Full mesh, (b) enlaryed view around cylinder.

analysis, ${ }^{33}$ around the onset of the primary and secondary instabilities of flow past a circular cylinder.

Our paper is outlined as follows: In Sec. II we outline the direct stability analysis method as well as discuss the direct optimal growth analysis needed to determine the singular values and their associated singular modes, which correspond to local convective instabilities. In Secs. III A I and III A 2 we discuss the results of the primarily instability obtained by considering two-dimensional and 3D perturbations about a steady flow state, substantiating the results in Refs. 28 and 29 . In Sec. III C we present results for the secondary instabilities about a periodic two-dimensional base flow.

\section{METHODOLOGY}

The fluid motion is govenned by the incompressible Navier-Stokes equations, written in nondimensional form as

$$
\begin{aligned}
& \partial_{t} \mathbf{u}=-(\mathbf{u} \cdot \nabla) \mathbf{u}-\nabla_{p}+\operatorname{Re}^{-1} \nabla^{2} \mathbf{u} \text { in } \Omega, \\
& \nabla \cdot \mathbf{u}=0 \text { in } \Omega,
\end{aligned}
$$

where $\mathbf{u}(\mathbf{x}, t)=[u, v, w](x, y, z, t)$ is the velocity field, $p(\mathbf{x}, t)$ is the kinematic (or modified) pressure field, and $\Omega$ is the flow domain, such as illustrated in Fig. I. In what follows we define Reynolds number as $\operatorname{Re}=U_{\infty} D / \nu$, with $U_{\infty}$ being the inflow velocity magnitude, $D$ the cylinder diameter, and $\nu$ the kinematic viscosity. In the present work all numerical computations of the base flows, whose two- and 3D energy growth characteristics we are interested in, will exploit the homogeneity in $z$ and require only a two-dimensional computational domain.

\section{A. Base flows and linear perturbations}

We first consider a base flow about which we wish to study the linear stability. The base flows for this problem are two-dimensional, time-dependent flows that therefore obey

$$
\partial_{t} \mathbf{U}=-(\mathbf{U} \cdot \nabla) \mathbf{U}-\nabla P+\operatorname{Re}^{-1} \nabla^{2} \mathbf{U} \text { in } \Omega,
$$


$\nabla \cdot \mathbf{U}=0$ in $\Omega$

where $P$ is defined in the associated base flow pressure. The boundary conditions imposed on $\mathrm{Eq}$. (2) are as follows: At the inflow and far field boundaries we impose a uniform velocity $U_{\infty}$, at solid walls we impose no-slip conditions, and at the downstream outflow boundary we impose a zero traction outflow boundary condition for velocity and pressure.

Our interest is in the evolution of infinitesimal perturbations $\mathbf{u}^{\prime}$ to the base flows. The linearized Navier-Stokes equations governing these perturbations are found by substituting

$$
\mathbf{u}=\mathbf{U}+\epsilon \mathbf{U}^{\prime} \text { and } p=P+\epsilon p^{\prime}
$$

where $p^{\prime}$ is the pressure perturbation, into the Navier-Stokes equations and keeping the lowest order (linear) terms in $\epsilon$. The resulting equations are as follows:

$$
\partial_{t} \mathbf{u}^{\prime}=-(\mathbf{U} \cdot \nabla) \mathbf{u}^{\prime}-\left(\mathbf{u}^{\prime} \cdot \nabla\right) \mathbf{U}-\nabla p^{\prime}+\operatorname{Re}^{-1} \nabla^{2} \mathbf{u}^{\prime} \text { in } \Omega,
$$

$$
\nabla \cdot \mathbf{u}^{\prime}=0 \text { in } \Omega \text {. }
$$

These equations are to be solved subject to appropriate initial and boundary conditions. The initial condition is an arbitrary incompressible flow which we denote by $\mathbf{u}_{0}$, i.e., $\mathbf{u}^{\prime}(\mathbf{x}, t=0)=\mathbf{u}_{0}(\mathbf{x})$. The boundary conditions we consider are simply homogeneous Dirichlet on all boundaries, i.e., $\mathbf{u}^{\prime}(\partial \Omega, t)=\mathbf{0}$. As discussed in Refs. 24 and 33 such homogeneous Dirichlet boundary conditions have the benefit of simplifying the treatment of the adjoint problem because they lead to homogeneous Dirichlet boundary conditions on the adjoint fields.

We note that the action of Eqs. (4a) and (4b) on an initial perturbation $\mathbf{u}^{\prime}(\mathbf{x}, 0)$ over time interval $\tau$ may be stated as

$$
\mathbf{u}^{\prime}(\mathbf{x}, \tau)=\mathcal{A}(\tau) \mathbf{u}^{\prime}(\mathbf{x}, 0)
$$

\section{B. LInear asymptotic stabillty analysls}

Through the eigensystem decomposition of this forward evolution operator $\mathcal{A}(\tau)$ we may determine the asymptotic stability of the base flow $\mathbf{U}$. In this case the solution is proposed to be the sum of eigenmodes,

$$
\mathbf{u}^{\prime}(\mathbf{x}, t)=\sum_{j} \exp \left(\lambda_{j} t\right) \tilde{\mathbf{u}}_{j}(\mathbf{x})+\text { c.c. }
$$

and we examine the eigenvalue problem

$$
\mathcal{A}(\tau) \tilde{\mathbf{u}}_{j}=\mu_{j} \tilde{\mathbf{u}}_{j}, \quad \mu_{j} \equiv \exp \left(\lambda_{j} \tau\right)
$$

If $\mathbf{U}$ is steady, then the largest eigenvalues $\mu_{j}$ of $\mathcal{A}(\tau)$ map to the eigenvalues $\lambda_{j}$ of largest real part for linear system (4), while the eigenmodes of $\mathcal{A}(\tau)$ and those of Eq. (4) are the same. If $\mathbf{U}$ is $T$-periodic then we set $\tau=T$ and consider this as a temporal Floquet problem, in which case the $\mu_{i}$ are Floquet multipliers and the eigenmodes of $\mathcal{A}(\tau)$ are the $T$-periodic
Floquet modes $\tilde{\mathbf{u}}_{j}(\mathbf{x}, t+T)=\tilde{\mathbf{u}}_{j}(\mathbf{x}, t)$ evaluated at a specific temporal phase. As noted in the introduction asymptotic instability analysis of unsteady flow past a circular cylinder has been applied by a number of authors, e.g., see Refs. 2 and 36.

\section{Optimal transient growth/singular value decomposition}

Our primary interest is in the energy growth of perturbations over a time interval, $\tau$, a parameter to be varied in the following studies. As is typical ${ }^{18}$ we define transient growth with respect to the energy norm of the perturbation flow, derived from the $L_{2}$ inner product

$$
2 E\left(\mathbf{u}^{\prime}\right)=\left(\mathbf{u}^{\prime}, \mathbf{u}^{\prime}\right) \equiv \int_{\Omega} \mathbf{u}^{\prime} \cdot \mathbf{u}^{\prime} d V
$$

where $E$ is the kinetic energy per unit mass of a perturbation, integrated over the full domain. If the initial perturbation $\mathbf{u}^{\prime}(0)$ is taken to have unit norm, then the transient energy growth over interval $\tau$ is

$$
\begin{aligned}
E(\tau) & =\left[\mathbf{u}^{\prime}(\tau), \mathbf{u}^{\prime}(\tau)\right] \\
& =\left[\mathcal{A}(\tau) \mathbf{u}^{\prime}(0), \mathcal{A}(\tau) \mathbf{u}^{\prime}(0)\right] \\
& =\left[\mathbf{u}^{\prime}(0), \mathcal{A}^{*}(\tau) \mathcal{A}(\tau) \mathbf{u}^{\prime}(0)\right],
\end{aligned}
$$

where we introduce $\mathcal{A}^{*}(\tau)$, the adjoint of the forward evolution operator in Eq. (5). The action of $\mathcal{A}^{*}(\tau)$ is obtained by integrating the adjoint linearized Navier-Stokes equations

$$
-\partial_{t} \mathbf{u}^{*}=-(\mathbf{U} \cdot \nabla) \mathbf{u}^{*}+(\nabla \mathbf{U})^{T} \cdot \mathbf{u}^{*}-\nabla p^{*}+\operatorname{Re}^{-1} \nabla^{2} \mathbf{u}^{*},
$$

$$
\nabla \cdot \mathbf{u}^{*}=0 \text { in } \Omega
$$

backward over interval $\tau$. The action of the symmetric component operator $\mathcal{A}^{*}(\tau) \mathcal{A}(\tau)$ on $\mathbf{u}^{\prime}$ is obtained by serial time integration of $\mathcal{A}(\tau)$ and $\mathcal{A}^{*}(\tau)$, i.e., we first use $\mathbf{u}^{\prime}(0)$ to initialize the integration of $\mathrm{Eq}$. (4) forward in time over interval $\tau$, then use the outcome to initialize the integration of Eq. (7) backward in time over the same interval.

The optimal perturbation is the eigenfunction of $\mathcal{A}^{*}(\tau) \mathcal{A}(\tau)$ corresponding to the compound operator's dominant eigenvalue, and so we seek the dominant eigenvalues $\lambda_{j}$ and eigenmodes $\mathbf{v}_{j}$ of the problem

$$
\mathcal{A}^{*}(\tau) \mathcal{A}(\tau) \mathbf{v}_{j}=\lambda_{j} \mathbf{v}_{j}
$$

Typically $G(\tau)$ is used to denote the maximum energy growth obtainable at time $\tau$, while the global maximum is denoted by $G_{\max }=\max _{\tau} G(\tau)$. Specifically,

$$
G(\tau)=\max _{j}\left(\lambda_{j}\right)=\max _{\mathbf{u}^{\prime} ! 0} \frac{\left[\mathbf{u}^{\prime}(\tau), \mathbf{u}^{\prime}(\tau)\right]}{\left[\mathbf{u}^{\prime}(0), \mathbf{u}^{\prime}(0)\right]} .
$$

We note that the eigenfunctions $\mathbf{v}_{j}$ correspond to right singular vectors of operator $\mathcal{A}(\tau)$, while their ( $L_{2}$-normalized) outcomes $\mathbf{u}_{j}$ under the action of $\mathcal{A}(\tau)$ are the left singular vectors, i.e., 


$$
\mathcal{A}(\tau) \mathbf{v}_{j}=\sigma_{j} \mathbf{u}_{j}
$$

where the sets of vectors $\mathbf{u}_{j}$ and $\mathbf{v}_{j}$ are each orthonormal. The singular values of $\mathcal{A}(\tau)$ are $\sigma_{j}=\lambda_{j}^{1 / 2}$, where both $\sigma_{j}$ and $\lambda_{j}$ are real and non-negative.

While long-time asymptotic growth is determined from the eigenvalue decomposition (6), optimal transient growth is described in terms of the singular value decomposition (9): The optimal initial condition and its (normalized) outcome after evolution over time $\tau$ are the right and left singular vectors, respectively, of the forward operator $\mathcal{A}(\tau)$ corresponding to the largest singular value, whose square (the largest eigenvalue of $\mathcal{A}^{*} \mathcal{A}$ ) is the optimal energy growth $G(\tau)$.

As already mentioned for an open flow, the most straightforward perturbation velocity boundary conditions to apply on both the inflow and outflow are homogeneous Dirichlet, i.e., $\mathbf{u}^{\prime}=\mathbf{0}$, for both the forward and adjoint linearized Navier-Stokes equations. The primitive variable, optimal growth formulation adopted in this work is discussed in further detail in Refs. 24 and 33 and follows almost directly from the treatments given by Refs. 37, 38, and 27 for strictly parallel or weakly nonparallel basic states.

\section{Time Integration and spatlal discretization}

Spectral $/ h p$ elements ${ }^{39}$ are used for spatial discretization, coupled with a Fourier decomposition in the homogeneous direction. Time integration is carried out using a velocity-conrection scheme. ${ }^{40.41}$ The same discretization and time integration schemes are used to compute base flows, and the actions of the forward and adjoint linearized NavierStokes operators. Base flows are precomputed and stored as data for the transient growth analysis. In the unsteady case, 32-64 time slices were stored and the base flow over one period of the evolution was reconstructed as required using Fourier interpolation.

The base flow was computed by enforcing a twodimensional symmetry for the calculation and time marching until the steady state is reached. In the case of flows that would otherwise be unstable, steadiness along the center line $y=0$ is enforced by setting $v=0$ and $\partial v / \partial y=0$ along that line.

Figure 1 shows the computational domain which has the spatial extent of $-8 \leq x / D \leq 95,-12.5 \leq y / D \leq 12.5$. The domain consists of 266 quadrilateral elements within which a polynomial order of $P=7$ was typically applied. For the steady flow computations we observe that this polynomial order is sufficient to resolve the eigenvalue up to three significant figures. The imposition of zero Dirichlet boundary conditions on the outflow for all perturbation computations necessarily means that there is a limit to the time interval over which the perturbation Eq. (4) can be integrated when the perturbation structure evolves within the wake. Conservatively assuming average velocity over the base flow wake of approximately $U_{\infty}$ we may consider integration time up to $T U_{\approx} / D \approx 100$ which roughly corresponds to 20 shedding cycles in the unsteady computations. This method of estimate agrees with accuracy tests done over shorter domains of $x / D \leq 25 D$ and $x / D \leq 40 D$.

\section{E. Three-dlmensional perturbatlons}

The final methodological issue to highlight is the treatment of 3D perturbations. Since the flow domain is homogeneous in the spanwise direction $z$, we can consider a Fourier modal expansion in $z$,

$$
\mathbf{u}^{\prime}(x, y, z)=\hat{\mathbf{u}}(x, y) \exp (i \beta z)+c \cdot c .
$$

where $\beta$ is the spanwise wavenumber. Linear systems (4) and (7) do not couple modes of different wavenumber, so modes for any $\beta$ can be computed independently. Since the base flow is such that $W(x, y)=0$ we may choose to work only with symmetric Fourier modes of the form

$$
\begin{aligned}
& u^{\prime}(x, y, z)=\hat{u}(x, y) \cos (\beta z), \\
& v^{\prime}(x, y, z)=\hat{v}(x, y) \cos (\beta z), \\
& w^{\prime}(x, y, z)=\hat{w}(x, y) \sin (\beta z), \\
& p^{\prime}(x, y, z)=\hat{p}(x, y) \cos (\beta z) .
\end{aligned}
$$

Such subspaces are invariant under Eqs. (4) and (7), and any eigenmode of $\mathcal{A}^{*}(\tau) \mathcal{A}(\tau)$ must be of form (10), or can be constructed by linear combination of these shapes. Decomposition (10) is used in practice because it is more computationally efficient than a full Fourier decomposition. An additional benefit is the elimination of eigenvalue multiplicity which exists for a full set of Fourier modes.

\section{RESULTS AND DISCUSSION}

\section{A. Steady flow}

We consider the two- and 3D transient growth characteristics about a two-dimensional steady flow. To appreciate how the transient growth characteristics compare to the linear stability of the flow, we consider two Reynolds numbers close to the primary two-dimensional linear instability.

Before considering the transient growth characteristics we recall that the asymptotic two-dimensional instability evolves through a Hopf bifurcation. The Hopf bifurcation arises at $\mathrm{Re}=46 \pm 1 .^{42,43}$ Below the critical Reynolds number at $\mathrm{Re}=45$, the leading perturbations obtained by linear stability analysis decays with a value of $\omega_{r}=-0.0024 \pm 1 \times 10^{-4}$ while the growth rate for the unstable flow at $\operatorname{Re}=50$ is $\omega_{r}=0.0188 \pm 1 \times 10^{-3}$, respectively. The associated eigenfunction of the perturbation causing the two-dimensional instability is shown in Fig. 3(b).

\section{Two-dimensional optimal perturbations}

Figure 2 compares the growth of the optimal energy as determined by the singular values of the linearized operator as a function of time span $\tau$ for the stable and unstable problems at $R e=45$ and $R e=50$. We observe that both the curve for $\mathrm{Re}=45$ and 50 recover the expected condition that the singular value at $\tau \rightarrow 0$ is $\lim _{\tau \rightarrow !} G(\tau)=1$ highlighting the point that at $\tau=0$ there is no relative growth. 


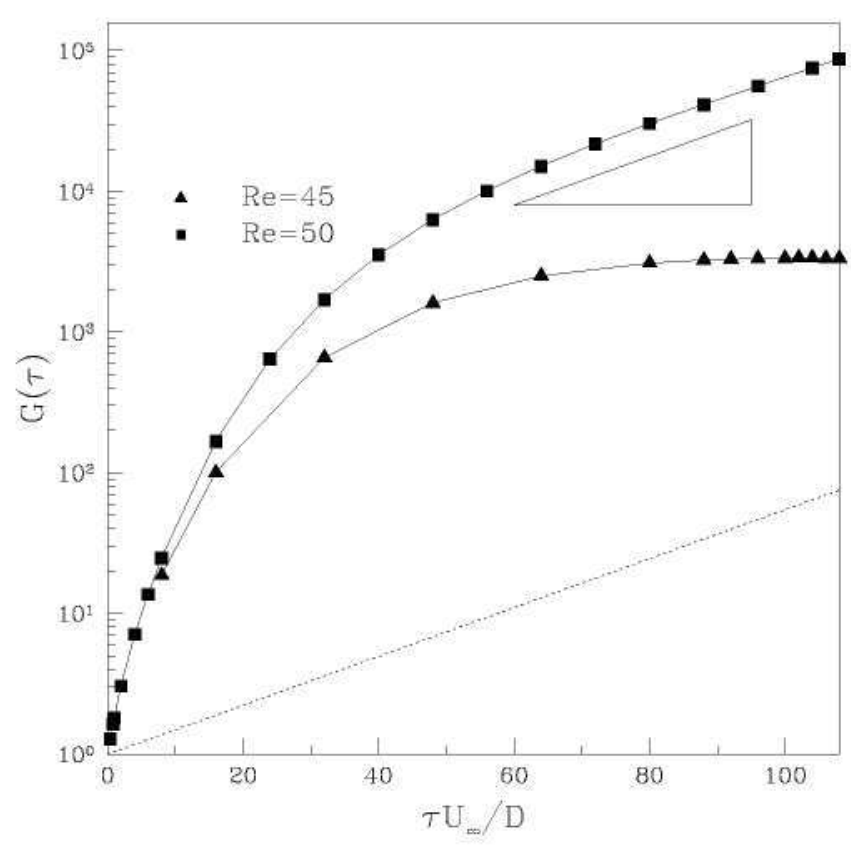

FIG. 2. Dependence of the leading singular values on integration time $T$ at Reynolds number of $\mathrm{Re}=45$ (triangles) and $\mathrm{Re}=50$ (squares) for twodimensional optimal growth on a steady two-dimensional base flow. Also indicated by the inset slope is the energy growth associated with the leading eigenmodes at Reynolds number $\operatorname{Re}=50$. Finally the dotted line denotes the relative energy growth rate associated with the leading linear eigenmode at $\operatorname{Re}=50$.

We recall that the physical interpretation of optimal growth, $G(\tau)$, is that in a predefined time $\tau$ the energy of the optimal perturbation (or right singular mode), will grow by a factor $G(\tau)$. We observe from Fig. 2 that the maximum energy growth of the optimal initial perturbation when $\operatorname{Re}=45$ is $G(\tau)=3357$ achieved at a time $\tau=102$. Investigations at a range of Re values indicate that the time to achieve maximum growth increases with Re up to the critical Reynolds number.

The curve therefore represents initial transient growth of over three orders of magnitude. As this growth is associated with the stable Reynolds number $\operatorname{Re}=45$ it eventually decays linearly with the rate equal to the negative eigenvalue obtained by a modal analysis.

Considering the optimal growth at $\mathrm{Re}=50$ in Fig. 2 we again see that the transient growth dominates at shorter times. However in this case the transient mechanism is then followed by exponential growth with a growth rate of $\omega_{r}=0.02$ known from the eigenmodal analysis. As indicated by the inset slope in Fig. 2 we observe that the slope of the curve asymptotically grows at a rate of the form

$$
\lim _{\tau \rightarrow \infty} G(\tau) \propto \exp ^{2 \omega_{r} \tau},
$$

where as we recall $\omega_{r}=0.02$ at $\operatorname{Re}=50$.

We emphasize that in both cases the transient behavior identified by computing the optimal growth analysis is still based on linear theory under the assumptions of Eq. (3). This (a)

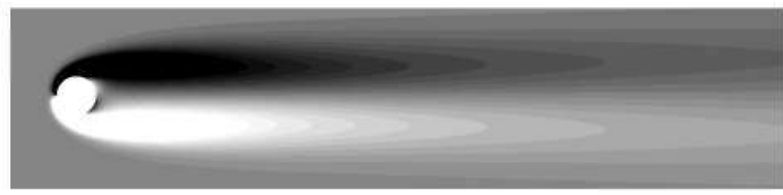

(b)

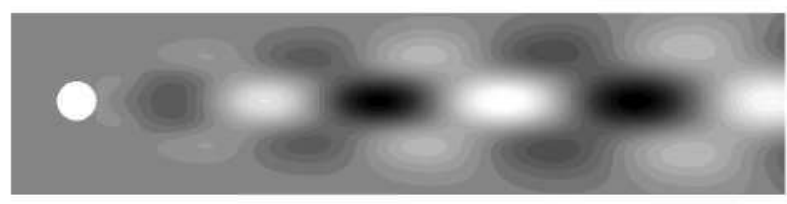

(c)

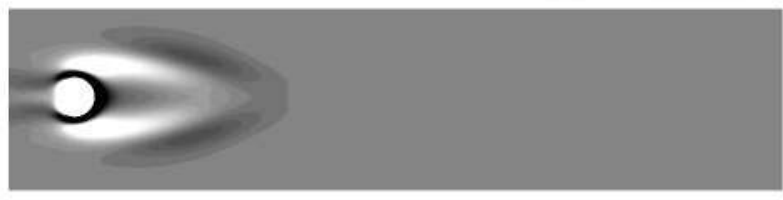

(d)

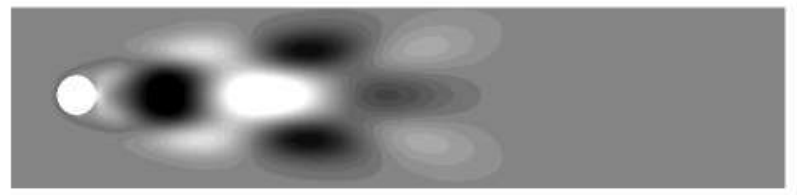

(e)

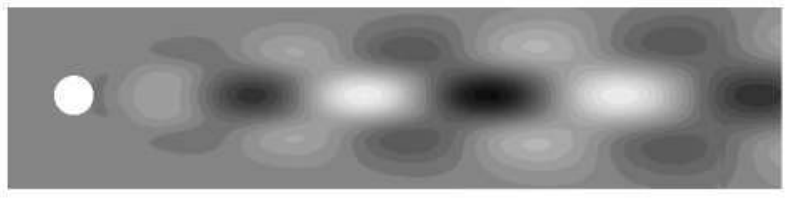

FIG. 3. Comparison of eigenmode with optimal transient growth modes: (a) Base flow spanwise vorticity at $\mathrm{Re}=50 \quad(20$ equispaced contours $-1 \leq \omega_{2} D / U \leq 1$ ), (b) spanwise vorticity of the leading eigenmode, (c) spanwise vorticity of the initial optimal growth mode over a time interval $\tau U_{\infty} / D=8$, (d) the associated spanwise vorticity of the final optimal growth mode associated with a time of $\tau U_{\infty} / D=8$, (e) spanwise vorticity of the final optimal growth mode associated with a time interval of $\tau U_{\infty} / D$ $=32$. In plots (b)-(e) we have used 20 equispaced contour levels over $-0.2 \leq \omega_{2} D / U \leq 0.2$

is consistent with the fact that the singular value analysis recaptures the exponential behavior for larger times. Our results agree with the transient analyses of plane Poiseuille flow of Schmid, ${ }^{19}$ who highlighted the process of transient growth for short times followed by a long-term exponential decay of stable basic states in parallel flows.

Figure 3 gives a comparison of the eigenmode and singular value analysis of the linear Navier-Stokes operator at $\operatorname{Re}=50$. We recall that Fig. 3(a) shows the vorticity of the base flow around the circular cylinder at this Reynolds number. Figure 3(b) then shows the vorticity of the leading eigenmode of the linearized operator. Ultimately the eigenmode evolves into the von-Kármán street and we recall that the leading eigenvalue at this Reynolds number is complex.

Figure 3(c) then shows the vorticity of the initial optimal growth mode associated with a relatively short integration time of $\tau=8$. We observe that the spatial distribution of the mode has a high energy concentration around the region of the boundary layer separation and in the near wake as also identified in the adjoint analysis of flow past a cylinder of Hill $^{28}$ and Gianetti and Luchini. ${ }^{29}$

In Fig. 3(d) we plot the final optimal growth mode associated with the time interval $\tau U_{\infty} / D=8$. This is the state into which the initial mode of Fig. 3(c) evolves at time $\tau U_{\infty} / D$ 


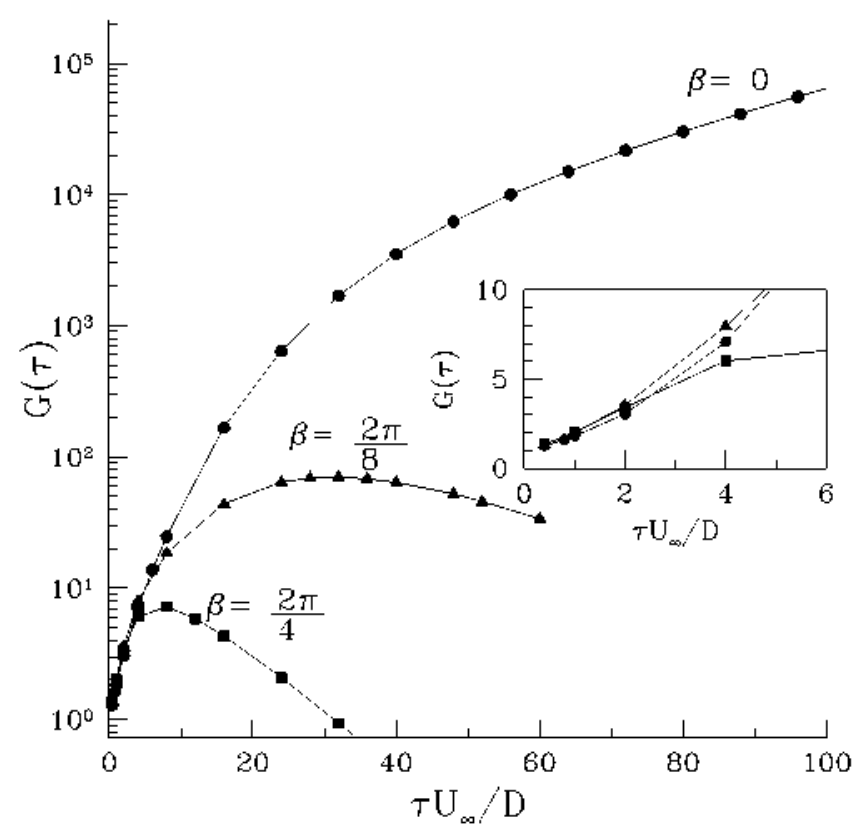

FIG. 4. Optinal growth $G(\tau)$ as a function of $\tau$ for spanwise wavenumbers $\beta=0,2 \pi / 4,2 \pi / 8$ at $\mathrm{Re}=50$. At longer time intervals the 3D perturbations eventually decay to zero. A short time intervals the $\beta \neq 0$ cases grow marginally faster than the $\beta=0$ case as indicated by the inset plot.

$=8$. At this time the associated energy of the right hand optimal modes has grown by a factor of $G(\tau)=24.76$ and evolved into the near wake region.

Finally in Fig. 3(e) we plot the final optimal growth mode associated with a longer time interval, $\tau U_{\mathrm{c}} / D=32$, at which time the optimal mode's energy has grown by a factor of $G(\tau)=1695$. As expected from our discussion of the optimal growth in Fig. 2 we observe that the optimal growth mode has evolved into the pattern (up to a change in sign) to that associated with the eigenmode of the linear stability analysis shown in Fig. 3(b).

\section{Three-dimensional perturbations}

Before discussing the results of the two-dimensional optimal growth we first consider 3D optimal growth perturbations on the two-dimensional base flow.

Figure 4 shows the singular values for different spanwise perturbations $L_{z} / D=4$ and $L_{z} / D=8$ at $\mathrm{Re}=50$. For the purposes of reference we have also included the twodimensional perturbation $(\beta=0)$ optimal growth rate previously shown in Fig. 2 . Three-dimensional eigenmode analysis about this base flow highlights that the flow is stable to three-dimensional perturbations consistent with the observation that the flow undergoes a two-dimensional Hopf bifurcation to the von-Kármán shedding mode. It is therefore not surprising that at larger time intervals the 3D optimal growths decay at a rate dictated by the slowest decaying eigenmode. For comparatively short time intervals $\tau<8$ however the 3D optimal growths are marginally higher although similar in magnitude to the $\beta=0$ case, as indicated by the inset in Fig. 4.

\section{B. Comparlson with nonllnear Navler-Stokes simulations}

As a validation of the transition mechanism we consider optimal growth modes as an initial condition for a nonlinear Navier-Stokes simulation. As an initial condition for these simulations we superpose the optimal growth mode for a fixed time interval $\tau U_{\infty} / D$ onto the steady state base flow, i.e., $\mathbf{u}(\mathbf{x}, 0)=\mathbf{U}(\mathbf{x})+\boldsymbol{\epsilon} \mathbf{u}^{\prime}(\mathbf{x}, 0)$, choosing $\epsilon$ small enough, $O\left(10^{-5}\right)$, so that we fulfill the assumptions of the perturbation ansatz.

In the following test we chose $L_{z}=4$ and the stable Reynolds number $\mathrm{Re}=45$ and monitor the perturbation energy $E(t)$ with time. As can be seen in Fig. 5, for all the time intervals considered, $\tau U_{\infty} / D=2,4,8,16$, and 32 there is a close agreement of the perturbation growth and the optimal growths (indicated by solid squares) evaluated using the transient growth analysis. We note that for a given time interval $\tau U_{\infty} / D$ the value of $G(\tau)$ does not correspond to the peak of the growth just the maximum achievable growth at the time point, $\tau U_{\infty} / D$. The value of $G(\tau)$ as a function of $t U_{\infty} / D$ does however represent the envelope of all optimal energy perturbations as shown in the last plot of Fig. 5.

\section{Discussion}

Consideration of the dotted line in Fig. 2 highlights the significant difference between the transient growth associated with the optimal initial condition and the exponential growth associated with the leading eigenmode of the operator. The dotted line represents an exponential energy growth associated with the unstable eigenvalue at $\mathrm{Re}=50$ with real component $\omega_{r}=+0.02$. From comparison of the curves in this plot we observe that at a time interval of $\tau U_{c} / D \approx 50$, the linear eigenmode leads to an energy growth of $O(10)$ while the optimal transient growth initial condition will experience a growth of $O\left(10^{3}\right)$ even in the subcritical flow at $\operatorname{Re}=45$. This reaffirms the assumption, based on findings in parallel flows, that there are nonmodal perturbations for the cylinder flow that grow significantly faster than the flow's eigenmodes over short time intervals.

For a linearly unstable base flow at $\operatorname{Re}=50$, this finding can also be interpreted in the following way: Upon perturbing the highlighted regions of the initial optimal modes in the near wake, the disturbances will grow algebraically over the interval, $\tau U_{\infty} / D \approx 32$, by a factor of order $10^{3}$. After this initial transient nonmodal growth the perturbation then contimues to evolve with an exponential form dictated by the leading eigenvalue of the flow to the two-dimensional timeperiodic state due to the linear instability.

Finally, we consider two distinct flow cases of a linear eigenmode and of an optimal growth mode. To achieve a kinetic energy growth by on order of magnitude at $\mathrm{Re}=50$, the eigenmode takes a time of $\tau \approx 58 D / U_{c o}$. To achieve the same growth, the optimal mode takes $\tau \approx 5 D / U_{\infty}$. This suggests a much more rapid route to transition to the saturated state of the primarily instability (vortex shedding). Having established the importance of transient growth modes and non-normality in this flow geometry, it remains an open question how these modes are initiated. We would suppose 

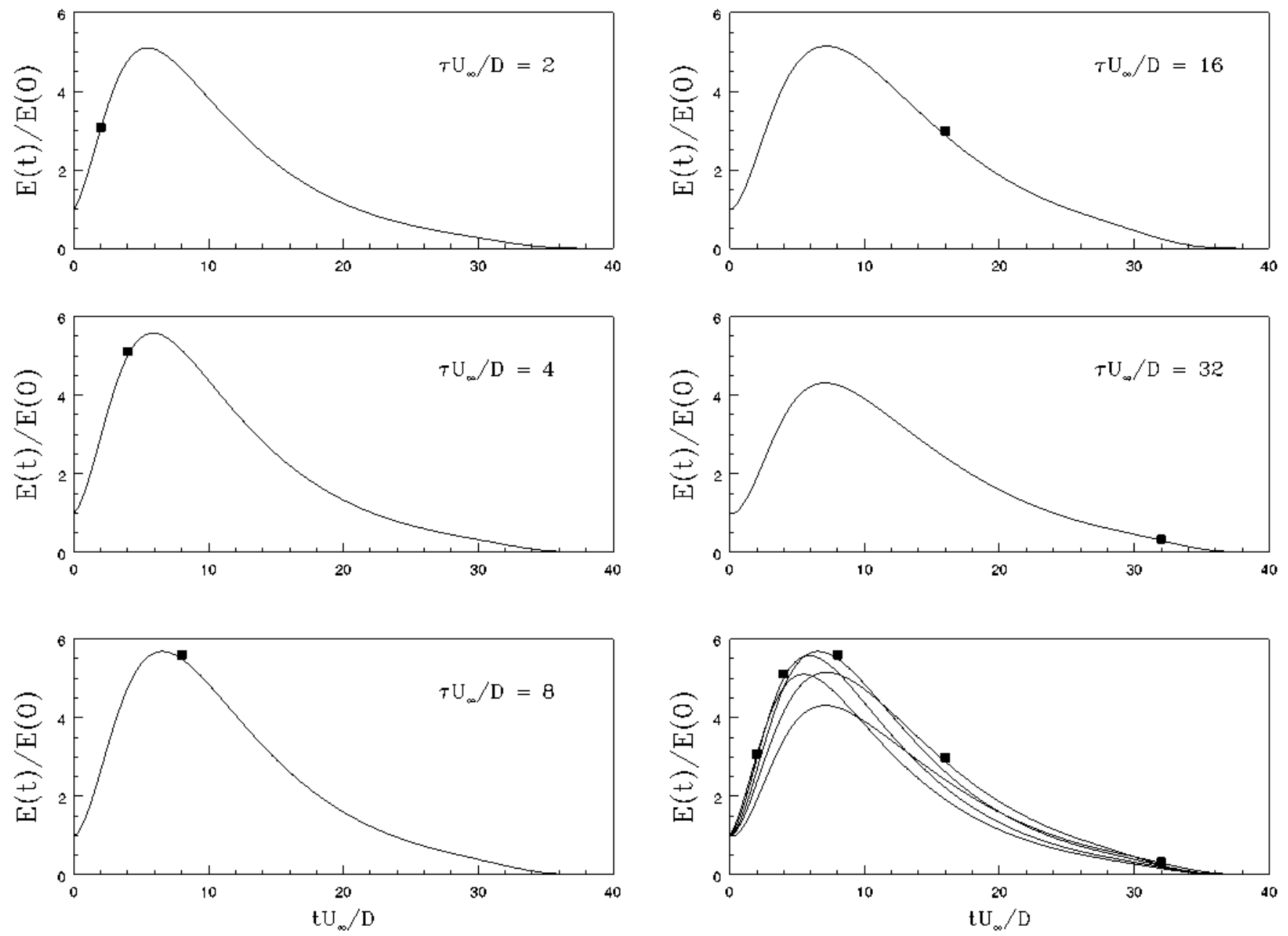

FIG. 5. Comparison of the relative energy growth of a Navier-Stokes simulation with $L_{x}=4$ initialized with a small $O\left(10^{-5}\right)$ perturbation of the initial optimal growth mode for different time intervals $\tau U_{\mathrm{co}} / D$. The squares indicate the point where the optimal mode achieves its optimal growth $G(\tau)$ at that time $t=\tau$. The last plot shows a superposition of all results where $G(\tau)$ is the envelope of all results.

that background noise might be sufficient to excite all modes, the system itself then selecting the optimal modes. Because the spatial location of the optimal growth initial condition is close to the cylinder, it makes it more likely that this mode is energized by perturbations on the cylinder, giving theoretical basis to the common practice of adding cylinder rotation to "kick start" the shedding process.

\section{Periodlc flow}

Analogous to the analysis of a steady base flow, the transient growth about a time-periodic base flow is a function of the time interval, $\tau$, the spanwise wavelength $L_{z}$ and the Reynolds number Re. However in the case of an unsteady or time-periodic base flow we have an additional parameter, $t_{0}$, which denotes the time phasing between the initiation of the transient growth mode and the base flow. In the following we will choose to restrict the parameter space by investigating the optimal transient growth about periodic states at two fixed Reynolds numbers of $\operatorname{Re}=200$ and $\operatorname{Re}=300$.

At $\operatorname{Re}=200$ the asymptotic stability analysis of the twodimensional periodic base flow using Floquet analysis identifies that the leading Floquet eigenmode has a wavelength of $L_{\bar{\tau}} \approx 4 D$ and is commonly referred to as mode $A^{2}$. In Ref. 2 the critical Reynolds number for the onset of this mode was determined to be $\operatorname{Re}_{\mathrm{cr}}=188.5$ when $L_{z}=3.96 \mathrm{D}$ and the transition is weakly subcritical. At this Reynolds number the next least stable mode has a shorter wavelength of $L_{z} \approx D$ and is referred to as mode $B$. Mode $B$ is stable at $\operatorname{Re}=200$ for all wavelengths. In the study of Barkley and Henderson, ${ }^{2}$ they determined that mode $B$ was a supercritical instability with a critical onset Reynolds number of $\mathrm{Re}=259$ at a wavelength of $L_{z}=0.82$. We therefore have chosen the second Reynolds number for our transient growth analysis to be at $\mathrm{Re}=300$ where both modes $A$ and $B$ are unstable.

\section{Determination of maximum time interval}

In the following investigations of an unsteady base flow we have adopted the mesh shown in Fig. 1 with an outflow at $x_{\text {cutflow }}=95 \mathrm{D}$. As with the transient growth analysis of the steady base flow we have applied zero Dirichlet conditions on all boundaries and so can only consider transient growth modes over time intervals where the final optimal mode does not reach the mesh boundaries. As we shall demonstrate the optimal initial condition of the transient growth mode is located near to the cylinder and so we therefore restrict our 

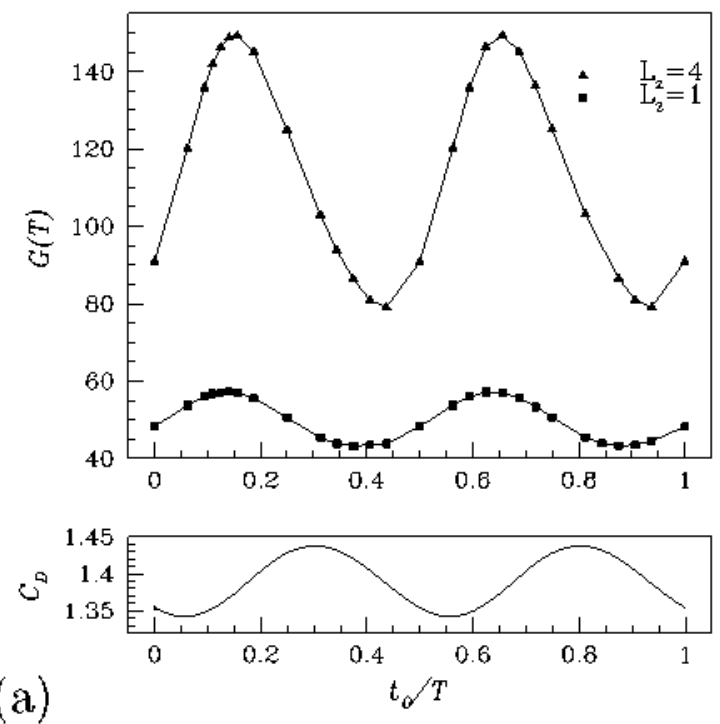

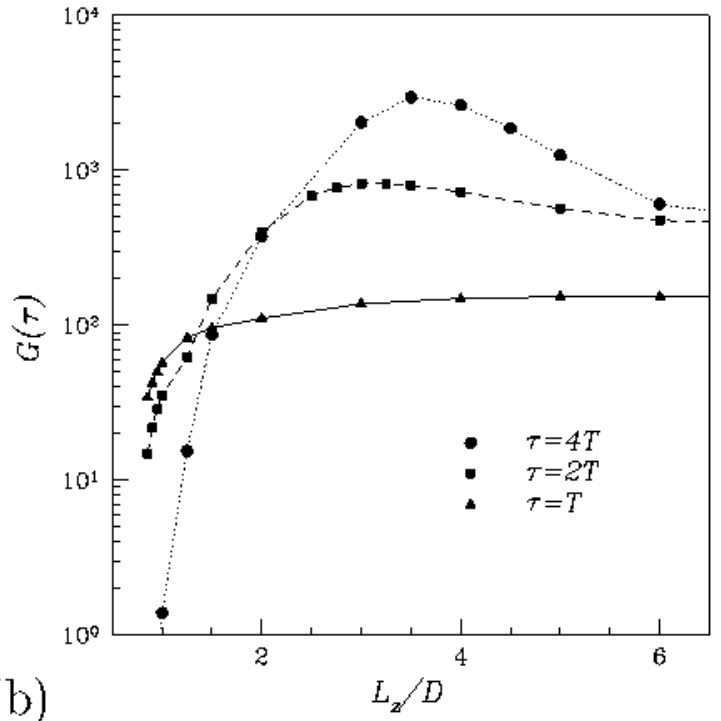

(b)

FIG. 6. Optimal transient growth $G(\tau)$ at $\operatorname{Re}=200$ : (a) Top plot shows the transient growth as a function of starting phase $t_{0}$ over one periodic shedding cycle $\tau=T$ for $L_{z}=1, L_{z}=4$. Botton plot shows the instantaneous drag coefficient of the base flow as a function of starting phase. (b) $G(\tau)$ evaluated at periodic final interval times $\pi / T=1,2$, and 4 as a function of $L_{i} / D$ for a starting phase of $t_{0} / T=0.156$.

attention to growths over a time interval of $\tau<x_{\text {outflow }} / U_{\infty}$ $=25$. For the Reynolds numbers considered this corresponds to approximately six shedding cycles.

\section{Three-dimensional optimal perturbations at $\mathrm{Re}=\mathbf{2 0 0}$}

The periodic two-dimensional base flow at $\mathrm{Re}=200$ is shown in Fig. 7(a). The Strouhal number at this Reynolds number was determined to be $T U_{c} / D=4.972$, where $T$ is the time for one shedding cycle. Before considering the spatial structure of the optimal transient growth modes we first investigate the influence of the phasing of the transient growth analysis with the base flow and the spanwise wavenumber $L_{\bar{\alpha}} / D=4$. In Fig. 6(a) we show the optimal growth $G(\tau)$ evaluated after one base flow shedding cycle, i.e., $\tau=T$ for different starting phases $t_{0} / T$. We observe that the variation of optimal transient growth has two peaks at approximately $t_{0} / T=0.15$ and 0.65 . The symmetry in time of this plot is to be expected given the spatiotemporal symmetry of the base flow and this is highlighted in the bottom plot of Fig. 6(a) where we show the instantaneous drag coefficient of the base flow as a function of starting phase. At this Reynolds number we observe that the peak starting phase arises approximately half way between the times of minimum to maximum instantaneous drag on the cylinder. We then fix the starting phase to $t_{0}=0.156 T$ which is near the first starting phase of maximum growth and consider the variation with spanwise wavenumber $L_{z} / D$, as shown in Fig. 6(b). In this figure we have considered three different time intervals of $\tau / T=1,2$, and 4 . We observe that for short spanwise wavelengths $L_{z} / D<1.25$ the optimal modes grow more rapidly at shorter time intervals. The maximum transient growth within the parameters considered arises for longer spanwise wavelengths at larger times. For $\tau=4 T$ we observe that the maximum value of $G(4 T)$ arises between $3.5 D<L_{\bar{c}}<4 D$.
In the above analysis, it was assumed that the peak at $t_{0}=0.156 T$ applies also when considering longer time intervals than $\tau / T=1$. As this is not necessarily the case, we have also considered the case when $L_{z} / D=4$ for time intervals up to $\tau / T=4$ and have not observed any changes on the maximum growth location of $t_{0}$. The associated time slice of the base flow at $t_{0}=0.156 \mathrm{~T}$ is shown in Fig. 7(a).

After consideration of the data shown in Fig. 6(b) we next consider the parameters $t_{0}=0.156 T, L_{z}=4$ for $\tau=T$ and $4 T$. At $L_{Z} / D=4$ and $\mathrm{Re}=200$ the Floquet multiplier is $\mu_{r}=1.115 \pm 0.005$. Figure 7 provides a comparison between the optimal transient growth modes and the Floquet eigenmode at $R e=200, L_{z}=4$. Figure $7(a)$ shows a snap shot of the base flow vorticity at $t_{0, \max }$ and Fig. 7 (b) highlights the spanwise vorticity pattern of mode $A$ at the same time instance. In Figs. $7(\mathrm{c})$ and $7(\mathrm{~d})$ we plot the initial optimum transient growth mode for $\tau=T$ and $\tau=4 T$, respectively. Note that the initial conditions are very similar in spatial structure up to an arbitrary scaling in sign. The initial conditions arise reasonably close to the cylinder and in the near wake. Finally Figs. 7 (e) and 7(f) show the associated final optimum transient growth modes for $\tau=T$ and $\tau=4 T$, respectively. The energy of the optimal modes has grown by a factor of $G(T)=149$ and $G(4 T)=2634$ for these two cases. We observe that increasing $\tau$ from $T$ to $4 T$ leads to a final state which is very similar to the Floquet mode shown in Fig. 7(b)

Figure 8 shows the optimal transient growth as a function of the time interval, $\tau$, for two spanwise wavelengths $L_{z}=1$ and $L_{z}=4$. Similar to the steady case shown in Fig. 2, transient growth can be observed within a few shedding cycles before the perturbation exhibits asymptotic exponential growth at a rate tending to that of the Floquet multiplier $\mu_{r}$. In this plot we have also indicated by the inset slope the growth rate of the Floquet analysis. Finally the inset slopes indicates the growth and decay rates from a purely "eigenmodal" (Floquet) initial condition. 
(a)

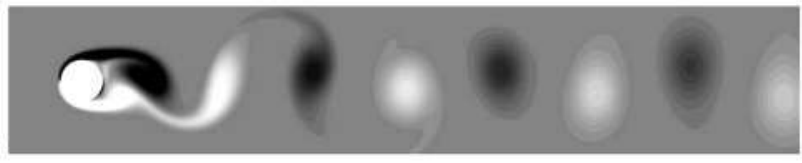

(b)

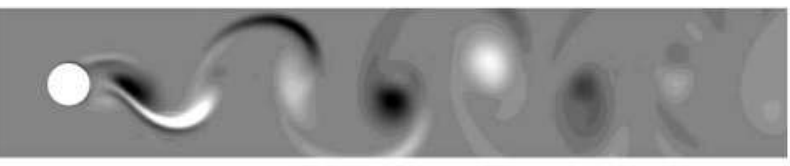

(c)

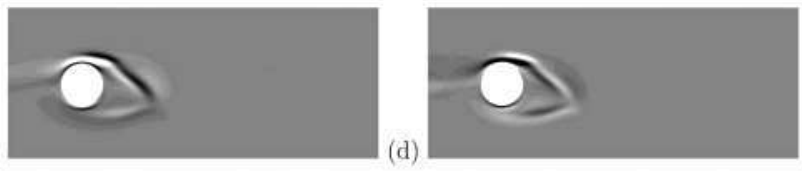

(e)

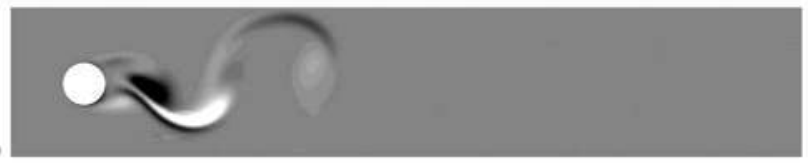

(f)

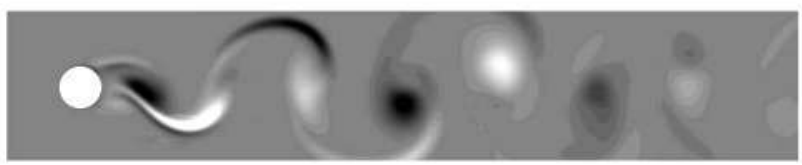

(g)
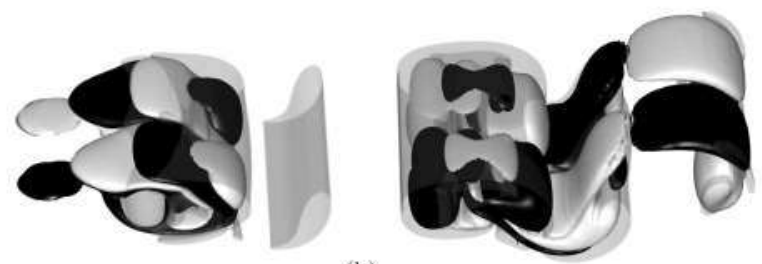

(h)

FIG. 7. (a) Spanwise vorticity at $\mathrm{Re}=200$ of the base flow at $t=0.1567$. Streamwise vorticity at $\mathrm{Re}=200$ : (b) Floquet eigenmode (mode $A$ ) when $L_{2} / D=4$, (c) initial optimum mode for $\tau=T$, (d) initial optimum mode for $\tau=4 T$, (e) final optimum mode $\tau=T$, and (f) final optimal mode for $\tau=4 T$. Plots (b)-(f) show 20 equispaced contours between $-0.08 \leq \omega_{x} D / U \leq 0.08$. Plots $(\mathrm{g})$ and $(\mathrm{h})$ show the $3 \mathrm{D}$ reconstructions of figure (c) and (e). The isosurfaces are of streamwise vorticity at $\pm 0,25$ (dark and light surfaces) and of spanwise vorticity at \pm 2 (translucent surfaces)

A similar analysis to that described in Sec. III B was undertaken to validate the evolution of the transient growth mechanism when using a nonlinear Navier-Stokes simulation starting from a small perturbation of the optimal transient growth mode. The growth histories at $L_{z}=1,4$ were observed to be in close agreement with those of the transient growth of the linearized equations.

\section{Three-dimensional optimum perturbations at $\mathrm{Re}=\mathbf{3 0 0}$}

We now turn our attention to the transient growth analysis at $\operatorname{Re}=300$, which is above the critical Reynolds number for mode $B$ to be asymptotically unstable. In Fig. 9 we again show the dependence of the optimal growth $G(\tau)$ as a function of the initial phase $t_{0}$ and the spanwise wavelength $L_{z}$. Figure 9(a) shows the variation of $G(\tau)$ for $\tau=T$ evaluated spanwise wavelengths of $L_{z}=0.85,1,3.5$, and 4 . We observe that for all the spanwise wavelengths considered the first maximum growth arises at an offset phase of $t_{0} / T=0.44$. Once again consideration of the bottom plot of Fig. 9(b) highlights that the maximum starting phase is associated with the instantaneous base flow drag going from its mini-

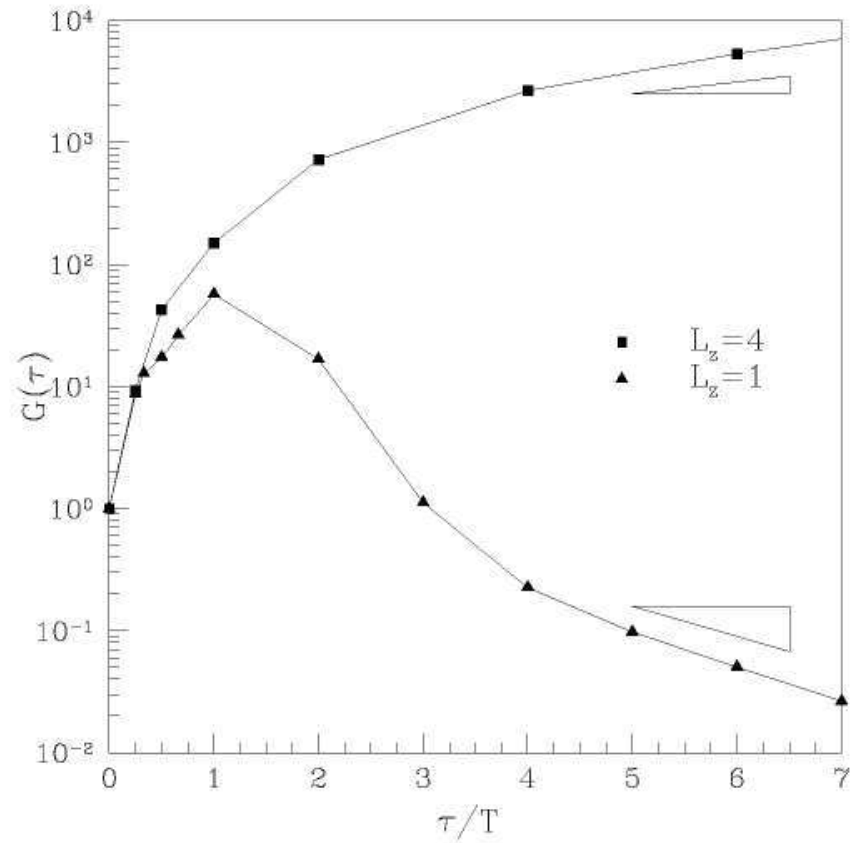

FIG. 8. Energy growth of the most growing unstable short, $L_{z}=1$, (triangles) and long, $L_{2}=4$, (squares) wavelength perturbations as a function of time $\tau / T$. The inset slope indicates the growth rate of the Floquet multiplier for a purely eigenmodal (Floquet) initial condition.

mum to maximum value during the shedding cycle. In Fig. 9(b) we consider a range of spanwise disturbances in the interval $0.33<L_{z}<6$ at three time intervals $\tau=T, 2 T, 4 T$ when $t_{0} / T=0.44$. For this Reynolds number we observe two local maxima at $L_{z} \approx 0.85$ and $L_{z} \approx 3.5$ reflecting modes $B$ and $A$, respectively, consistent with the Floquet analysis. For these flow parameters we deduce that both the long (mode $A$ ) as well as the short (mode $B$ ) wavelength optimal growth modes grow exponentially after an initial transient phase. At this Reynolds number of the Floquet multiplier of mode $A$ at $L_{z}=3.5$ is $\mu_{r}=1.9$ and the Floquet multiplier of mode $B$ at $L_{z}=0.85$ is $\mu_{r}=2.13$. The larger magnitude of the Floquet multiplier implies that the asymptotic behavior establishes itself after only two shedding cycles. Similar to the results presented in Fig. 7, a snap shot of the base flow as well as the associated most unstable mode $B$ is shown in Figs. 10(a) and 10 (b), respectively. The evolution of the initial optimum mode into mode $B$ is analogous to the previous scenario where only mode $A$ is unstable. In Figs. 10 (c) and 10 (d) we show the spatial structure in terms of spanwise vorticity of the optimal transient growth modes at $\tau=T$ and $\tau=2 T$, respectively. Finally in Figs. 10(e) and 10(f) we show the final state of the optimal modes for $\tau=T$ and $\tau=2 T$, which have undergone a growth of $G(T)=271$ and $G(2 T)=2143$, respectively. We note that optimal modes are only determined up to an arbitrary change in sign.

\section{Discussion}

Similar to the steady base flow study in Sec. III A, in the case of periodic base flow at Reynolds number above the onset of asymptotic instability we observed a close connection between the optimum transient growth mode and the exponentially growing eigenmode of the linearized system. 

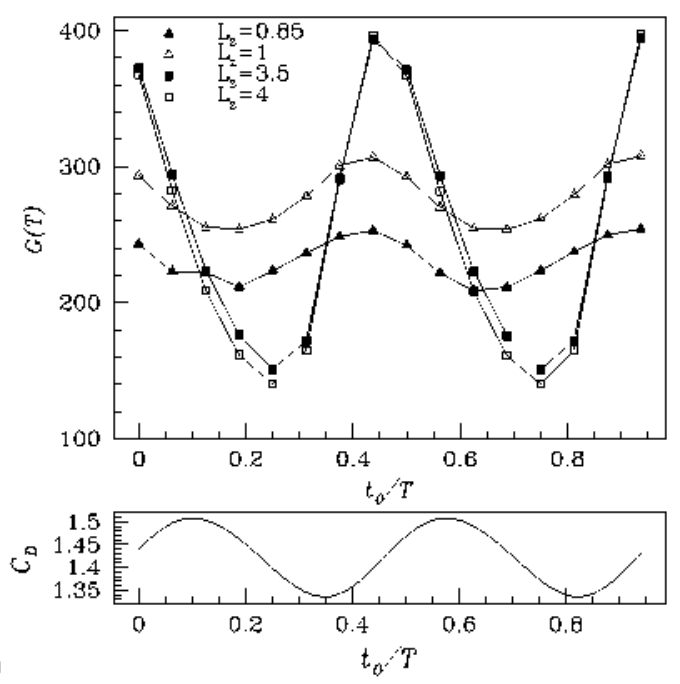

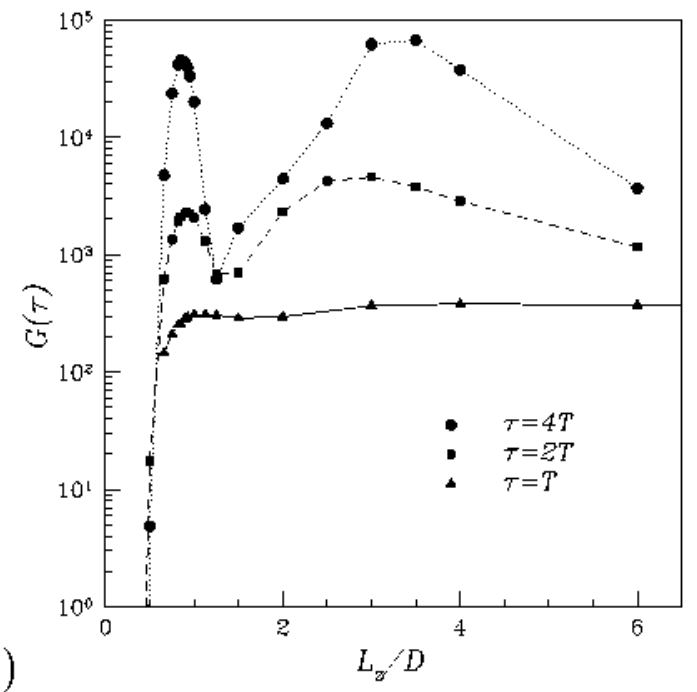

(b)

FIG. 9. Optimal transient growth $G(\tau)$ at $\mathrm{Re}=300$ : (a) Top plor shows the transient growth as a function of starting phase $t_{0}$ over one periodic shedding cycle $\tau=T$ for $L_{2} / D=0.85 .1 .3 .5$. and 4. Bottom plot shows the instantaneous drag coefficient of the base fow as a function of starting phase. (b) $G(\tau)$ evaluated at periodic final interval times $\pi / T=1$. 2. and 4 as a function of $L_{z} / D$ for a starting phase of $t_{0} / T=0.44$

At the Reynolds numbers considered we observe that as the transient growth process evolves the spatial structure of the optimal growth modes (left singular mode) aligns itself to the leading eigenmode of the linearized operator and it is through this mode that the flow transitions to a $3 \mathrm{D}$ unsteady flow state.

One can compare the transition routes via transient growth and asymptotic eigenmodal growth. We recall that at $\mathrm{Re}=200, L_{z}=4$ the Floquet multiplier has a magnitude $\mu_{r}=1.12$. Therefore as highlighted in Fig. 8 the asymptotic (Floquet) instability will grow one order of magnitude in approximately ten shedding cycles, as opposed to three orders of magnitudes when considering the energy growth through a transient process. However the relative time frame of the transient and asymptotic behavior necessarily depends on the strength of the asymptotic growth. Therefore at $\mathrm{Re}$ $=300$ where the Floquet multiplier for mode $B$ was twice as large we observe that the transient growth mechanism has already aligned itself to mode $B$ eigenmode within two shedding cycles as opposed to four shedding cycles for the transient growth mode to align itself to mode $A$ at $\operatorname{Re}=200$.

We have observed that when the flow is asymptotically unstable the optimal growth mode and eigenmode can become aligned. However we reiterate that this does not mean that we require asymptotic instability to observe transient growth. We recall that in Fig. 6 at $\operatorname{Re}=200, L_{z}=1$ the flow is asymptotically stable but transient growth mechanisms are still observed. Although in this case the growth was relatively small, larger transient growths have been reported for asymptotically stable flows past a backward-facing step ${ }^{24}$ and steady and pulsatile flows in stenotic geometries. ${ }^{25}$ The possibility of large transient growths in asymptotically stable flows highlights the need to distinguish two different notions of sensitivity versus (in)stability. The idea of sensitivity, as investigated in Refs. 28 and 29 is related to optimal control theory and the adjoint of the linear operator.

\section{E. Navier-Stokes simulation at $R e=200, L_{z}=4$}

The Navier-Stokes simulations discussed in Sec. III B and analogous tests (not reported here) for the periodic base flows were performed under near linear conditions. In these tests we allowed only the mean mode and one spanwise wavelength to develop in order to show coherence between the obtained transient growth values and the temporal growth of the identified singular perturbations. In the following, we employ a full Navier-Stokes simulation allowing for nonlinear effects due to interaction of high harmonics of spanwise Fourier modes. This enables us to investigate the full path to transition.

Two simulations were performed both at a spanwise wavelength of $L_{z}=4$ with 16 Fourier modes at a polynomial order of 7 . In the first simulation, we superimposed the Floquet mode $A$ onto a base flow at $\operatorname{Re}=200$ while in the second simulation the initial optimum growth mode based on a growth interval of $\tau=4 T$ was superimposed on the twodimensional base flow. Both initial disturbances were scaled to have an initial energy magnitude of approximately $10^{-12}$, small enough to satisfy all linear assumptions.

Figure 11 summarizes the DNS study for initial conditions based on both the optimum growth mode as well as the eigenmode obtained by Floquet analysis. In this figure we plot the energy evolution over nondimensional time of the mean mode and the first three spanwise Fourier harmonics. The solid lines denote the energy growth from the optimal growth initial conditions while the dashed lines denote the growth from the Floquet initial conditions. Similar to the energy growth comparison in Fig. 8, the transient growth exceeds the exponential growth by approximately three orders of magnitudes within a few shedding cycles before they both evolve exponentially. As demonstrated by the inset slope the $K=1$ mode then grows at the rate estimated by the Floquet analysis (i.e., $\mu_{r}=1.115$ ). The inset plot shows the 


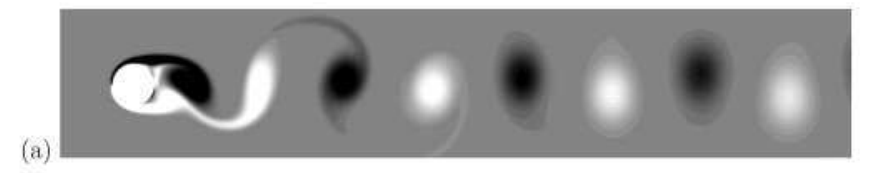

(b)

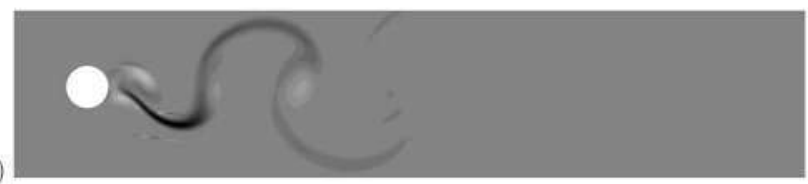

(c)

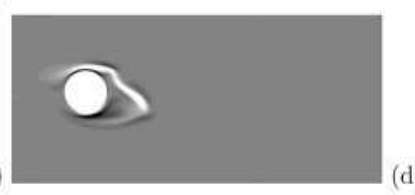

(e)
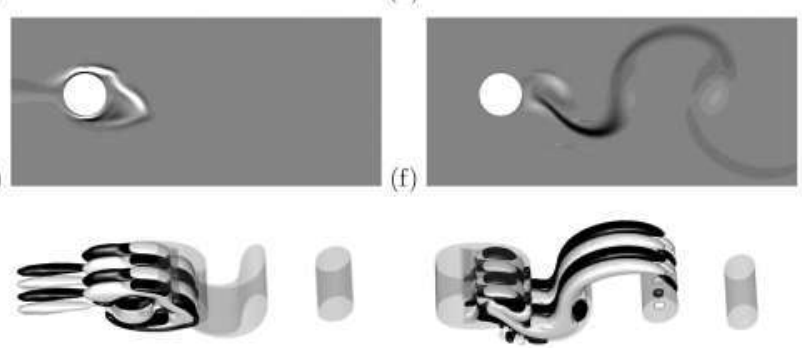

(g)

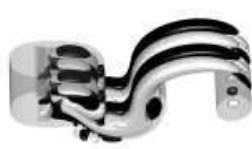

(h)

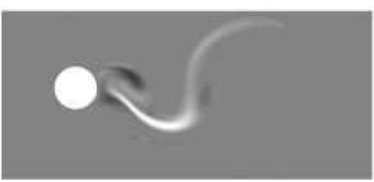

FIG. 10. (a) Spanwise vorticity at $\operatorname{Re}=300$ for the base flow $t=0.44 T$. Streamwise vorticity at $\mathrm{Re}=300$ : (b) Floquet eigenmode (mode $B$ ) when $L_{z}=0.85$, (c) initial optimum mode for $\tau=T$, (d) final optimum mode for $\tau=T$, (e) initial optimum mode for $\tau=2 T$, and (f) final optimal mode for $\tau=2 T$. Plots (b)-(f) show 20 equispaced contours between $-0.2 \leq \omega_{x} D / U$ $\leq 0.2$. Plots $(\mathrm{g})$ and $(\mathrm{h})$ show the initial and final modes for $\tau=T$ with 20 times the perturbation superimposed on the base flow. The isosurfaces are of streamwise vorticity at \pm 0.25 (dark and light surfaces) and of spanwise vorticity at \pm 2 (translucent surfaces).

growth of the $K=1$ mode with optimal initial conditions over a shorter time interval. We note that the $K=0$ mode is influenced by the emergence of the high frequency modes but on the logarithmic-linear scale of this plot this effect is not visible.

In line with our optimal growth analysis and NavierStokes simulations allowing for one spanwise wavelength to develop, the results confirm the connection between optimal growth modes and (Floquet) eigenmodes. The simulation shows that the evolution to a developed 3D state is accelerated by perturbing the regions in the near wake, the optimal growth modes, accounting for a more rapid transition scenario than that expected by linear theory based on eigenmodal analysis where growth typically is promoted in the far wake.

We find modes that have, over a short initial time period, significantly faster growth through a transient growth mechanism than the exponential growth of the leading eigenmode instability. These modes do eventually, for longer times collapse into 3D eigenmodes. However, indicating them solely by their growth rate would hide the fact that the growth is orders of magnitude higher than purely modal growth at any given time, as seen in Fig. 11. The transient growth final mode collapses to the eigenmode, but involves a different initial condition, that results in orders of magnitude higher

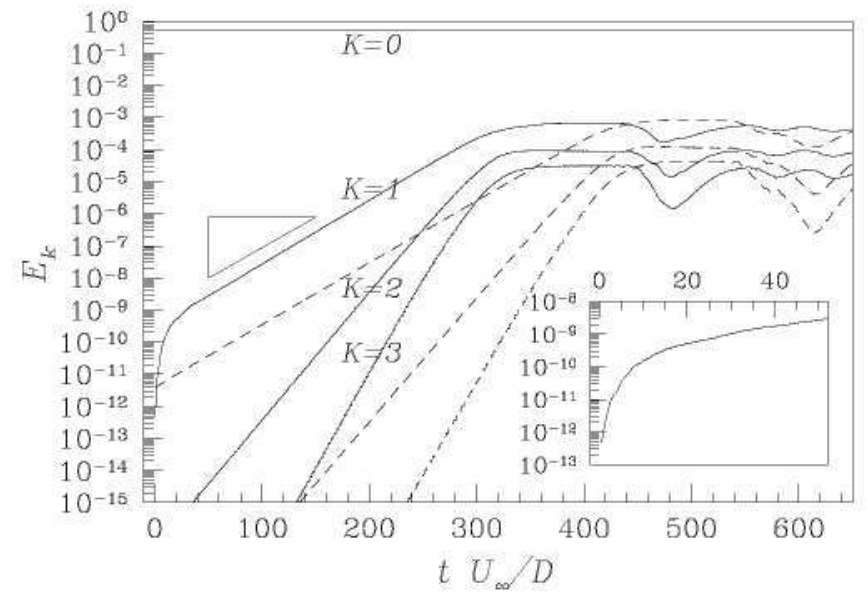

FIG. 11. DNS of the flow past a cylinder at $\mathrm{Re}=200$ and spanwise extent of $L_{z}=4$. The dashed curves were obtained by superposing the Floquet mode onto the periodic base state, the solid curves were computed by superposition of an initial optimal growth mode onto the same base flow. Comparison of energy growth of the mean $(K=0)$ and first three spanwise Fourier modes $(K=1,2,3)$. The inset slope indicates the growth rate of the Floquet analysis and the inset plot shows the growth of the optimal mode over a shorter time period.

growth at a given time than the purely modal initial condition. Thus transition is achieved much faster by this route than by simply modal growth.

\section{CONCLUSIONS}

Direct optimum growth analysis has been applied to investigate the transient nature of nonmodal perturbations in flow past a circular cylinder. To quantify the transient growth, we have computed the leading singular values of the linearized Navier-Stokes evolution operator by exploiting matrix-free eigenvalue analysis combined with the favorable approximation properties of the spectral $/ h p$ element method.

Initially we considered the linearized stability about a two-dimensional steady base flow at $\operatorname{Re}=45$ and $\operatorname{Re}=50$. The analysis demonstrated that the two-dimensional optimum growth modes appear in the near wake of the cylinder and the energy of these modes are amplified to the order of $10^{3}$ over a time interval of $U_{\infty} \tau / D \approx 30$. This optimal mode has a significantly larger growth than the unstable eigenmode at $\mathrm{Re}=50$ over this time period. At $\mathrm{Re}=50$ and for larger time intervals the optimal growth mode becomes aligned to the eigenmodal growth associated with the von-Kármán street. The identification of the optimal growth mechanism provides a more complete description for the transition from steady to periodic flow where fast initial transient growth precedes the exponential growth of an eigenmodal mechanism.

A analogous analysis has also been applied to the linearized flow about periodic base flows at $\mathrm{Re}=200$ and 300. At these Reynolds numbers the flow is known to be threedimensionally linearly unstable for wavelengths of $L_{z} \approx 4$, the so-called mode $A$ instability, and $L_{z} \approx 1$, for the so-called mode $B$ instability. Once again we have observed a connection between modal and nonmodal (transient) perturbations. A transient growth in energy of up to three orders of magnitude arises between two to four shedding cycles. This tran- 
sient growth again precedes the eigenmodal mechanisms and subsequent transition to a 3D flow state. Similar to the steady flow case the initial optimal perturbation arise in the near wake region of the separated shear layers. Simulations of the nonlinear 3D Navier-Stokes equations confirms the analysis.

The connection between transient growth and convective instability has previously been made for the GinzbergLandau equations by Cossu and Chomaz. ${ }^{21}$ This connection has been further amplified in more recent papers. ${ }^{22,44,24,25}$ However in most of these studies, which have primarily considered steady base flows, the connection of transient growth and convective instability was reinforced though the identification of the convective growth of a small perturbation which maintained a similar spatial structure. However in the current problem, particularly when the base flow is unsteady, we are unable to identify a "convective" structure in order to reinforce the direct association of the transient growth mechanism with convective instability. We note, however, that local sectional analyses of the wake profiles of steady flow behind a cylinder ${ }^{43,46}$ have identified that a region of absolute instability exists behind the cylinder followed by region of convective instability for $\mathrm{Re}>20$. It is not unreasonable therefore to connect the transient growth at $\operatorname{Re}=45$ considered in Sec. III A with an amplification through this region of absolute local instability followed by subsequent decay further downstream.

The analysis presented nevertheless quantifies the transient growth mechanism and allows inspection of its spatial structure. Furthermore, the amplification in the unstable case results in something like - and is therefore coupled with - an unstable eigenmode, leading to much faster transition than via the purely modal route. It is therefore reasonable to consider the analysis as presenting an accelerator to an existing mechanism. Although it is not clear how to energize the initial state of the optimal growth modes, from a control point of view it seems plausible that these mechanisms are attractive due to their spatial localization near to the cylinder.

\section{ACKNOWLEDGMENTS}

The authors would like to acknowledge Hugh Blackburn and Dwight Barkley for their contribution into the Transient Growth problem formulation and Bruno Carmo for his help in validating the results. The authors would also like to thank the anonymous referees for helpful comments regarding the connection between the transient growth mechanism and the convective instability. A. Sharma wishes to acknowledge support under EPSRC Grant EP/E017304/1. S.J.S. would like to acknowledge support from an EPSRC Advanced Research Fellowship.

Partial support has been received by the Air Force Office of Scientific Research, under Grant No. F49620-03-1-0295 to nu-modeling S.L., monitored by Dr. T. Beutner (now at DARPA), Lt. Col. Dr. R. Jefferies and Dr. J. D. Schmisseur of AFOSR and Dr. S. Surampudi of the European Office of Aerospace Research and Development.

\footnotetext{
'P. Drazin and W. Reid, Hydrodynamic Stability (Cambridge University Press, Cambridge. 1981)

${ }^{2}$ D. Barkley and R. Henderson, "Three-dimensional floquet stability analy-
}

sis of the wake of a circular cylinder." J. Fluid Mech. 322. 215 (1996). ${ }^{3}$ L. Tuckerman and D. Barkley, in Numerical Methods for Bifurcation Problems and Large-Scale Dynamical Systems, edited by E. Doedel and L. Tuckerman (Springer, New York, 2000), Vol. 119, pp. 466-543.

${ }^{4} \mathrm{~V}$. Theofilis, "Advances in global linear instability of nonparallel and three-dimensional flows," Prog. Aerosp. Sci. 39, 249 (2003).

${ }^{5} \mathrm{U}$. Ehrenstein, "On the linear stability of channel flows over riblets." Phys. Fluids 8, 3194 (1996).

${ }^{6} \mathrm{Y}$. Ding and M. Kawahara, "Linear stability of incompressible flow using a mixed finite element method," J. Comput. Phys. 139, 243 (1998).

${ }^{7}$ H. M. Blackburn, "Three-dimensional instability and state selection in an oscillatory axisymmetric swirling flow." Phys. Fluids 14, 3983 (2002).

${ }^{8}$ V. Theofilis, A. Fedorov, D. Obrist, and U. C. Dallmann. "The extended Görtler-Hänmerlin model for linear instability of three-dinensional inconpressible swept attachment-line boundary layer flow," J. Fluid Mech. 487, $271(2003)$.

${ }^{9} \mathrm{~V}$. Theofilis. P. W. Duck, and J. Owen, "Viscous linear stability analysis of rectangular duct and cavity fows," J. Fluid Mech. 505. 249 (2004).

${ }^{10} \mathrm{~S}$. J. Sherwin and H. M. Blackburn, "Three-dimensional instabilities and transition of steady and pulsatile flows in an axisymmetric stenotic tube," J. Fluid Mech. 533. 297 (2005)

${ }^{11} \mathrm{~L}$. González, V. Theofilis. and R. Gómez-Blanco. "Finite element methods for viscous incompressible biglobal instability analysis on unstructured meshes," AIAA J. 45. 840 (2007).

${ }^{12} \mathrm{H}$. M. Blackburn and S. J. Sherwin, "Instability modes and transition of pulsatile stenotic fow: Pulse-period dependence," J. Fluid Mech. 573, 57 (2007).

${ }^{13} \mathrm{~V}$. Theofilis, S. Hein, and U. Dallmann, "On the origins of unsteadiness and three-dimensionality in a laminar separation bubble," Philos. Trans. R. Soc. London. Ser. A 358, $3229(2000)$.

${ }^{14} \mathrm{R}$. Henderson and D. Barkley, "Secondary instability in the wake of a circular cylinder," Phys. Fluids 8, 1683 (1996).

${ }^{15} \mathrm{M}$. Schatz, D. Barkley, and H. Swinney, "Instabilities in spatially periodic: channel tow," Phys. Fluids 7, 344 (1995).

${ }^{16} \mathrm{~K}$. M. Butler and B. F. Farrell, "Three-dimensional optimal perturbations in viscous shear flow." Phys. Fluids A 4, 1637 (1992).

${ }^{17}$ L. N. Trefethen, A. E. Trefethen, S. C. Reddy, and T. Driscoll. "Hydrodynamic stability without eigenvalues," Science 261. 578 (1993).

${ }^{18} \mathrm{P}$. Schmid and D. Henningson, Stability and Transition in Shear Flow's (Springer, 2001).

${ }^{19}$ P. J. Schmid. "Nonmodal stability theory." Annu. Rev. Fluid Mech. 39. 129 (2007).

${ }^{20} \mathrm{~B}$. F. Fartell. "Optimal excitation of perturbations in viscous shear flow," Phys. Fluids 31. 2093 (1988).

${ }^{21} \mathrm{C}$. Cossu and J. M. Chomaz, "Global measures of local convective instabilities," Phys. Rev. Lett. 78, 4387 (1997).

${ }^{22}$ J.-M. Chomaz, "Global instabilities in spatially developing flows: Nonnormality and nonlinearity." Annu. Rev. Fluid Mech. 37, 357 (2005).

${ }^{23} \mathrm{O}$. Marijuet. D. Sipp, and L. Jacquin, "Global optimal perturbations in a separated flow over a backward-rounded-step," 36th AIAA Fluid Dynamics Conference and Exhibit, San Francisco, CA, USA, 2006, Paper No. 2006-2879.

${ }^{24} \mathrm{H}$. M. Blackburn, D. Barkley, and S. J. Sherwin, "Convective instability and transient growth in flow over a backward-facing step," J. Fluid Mech. 603. $271(2008)$.

${ }^{25} \mathrm{H}$. Blackburn, S. J. Sherwin, and D. Barkley, "Convective instability and transient growth in steady and pulsatile stenotic flows," J. Fluid Mech. 607, 267 (2008).

${ }^{26} \mathrm{U}$. Ehrenstein and F. Gallaire. "On two-dimensional temporal modes in spatially evolving open flows: the flat-plate boundary layer," J. Fluid Mech. 536. 209 (2005).

${ }^{27}$ J. Hopffner, L. Brandt. and D. S. Henningson. "Transient growth on boundary layer streaks," J. Fluid Mech. 537. 91 (2005).

${ }^{28} \mathrm{D}$. Hill, "A theoretical approach for analyzing the restabilization of wakes," 30th AIAA Aerospace Sciences and Exhibit. Reno. NV. USA. 1992, Paper No. 1992-0067.

${ }^{29} \mathrm{~F}$. Giannetti and P. Luchini, "Structural receptivity of the first instability of the cylinder wake," J. Fluid Mech. 581, 167 (2007).

${ }^{30}$ D. Barkley, M. G. M. Gomes, and R. D. Henderson, "Three-dimensional instability in flow over a backward-facing step." J. Fluid Mech. 473, 167 (2002).

${ }^{31}$ H. M. Blackburn and J. M. Lopez, "The onset of three-dimensional standing and modulated travelling waves in a periodically driven cavity flow," J. Fluid Mech. 497. 289 (2003). 
${ }^{32}$ J. R. Elston, H. M. Blackburn, and J. Sheridan. "The primary and secondary instabilities of fow generated by an oscillating circular cylinder," $J$. Fluid Mech. 550. 359 (2006).

${ }^{33}$ D. Barkley. H. M. Blackburn, and S. J. Sherwin. "Direct optimal growth analysis for timesteppers." Int. J. Numer. Methods Fluids 57. 1435 (2008).

${ }^{34}$ A. Sharma, N. Abdessemed, S. Sherwin, and V. Theofilis, "Optimal growth modes of tows in complete geometries." IUTAM Symposium on Flow Control and MEMS, London. UK, 2006.

${ }^{35} \mathrm{C}$. H. K. Williamson. "The existence of two stages in the transition to three-dimensionality of a cylinder wake," Phys. Fluids 31, 3165 (1988).

${ }^{36} \mathrm{H}$. M. Blackburn and J. M. Lopez, "On three-dimensional quasi-periodic Floquet instabilities of two-dimensional blutf body wakes." Phys. Fluids 15, L57 (2003).

${ }^{37} \mathrm{P}$. Corbett and A. Bottaro, "Optimal perturbations for boundary layers subject to stream-wise pressure gradient," Phys. Fluids 12, 120 (2000).

${ }^{38} \mathrm{P}$. Luchini, "Reynolds-number-independent instability of the boundary layer over a flat surface: optimal perturbations," J. Fluid Mech. 404, 289 $(2000)$.
${ }^{39}$ G. E. Karniadakis and S. J. Sherwin. Spectral/hp Element Methods for $C F D$ (OUP, New York, 1999)

${ }^{40} \mathrm{G}$. Karniadakis, M. Israeli, and S. Orszay, "High-order splitting methods for the incompressible Navier-Stokes equations." J. Comput. Phys. 97. 414 (1991).

${ }^{41}$ J. S. J. L. Guermond, "A new class of truly consistent splitting schemes for incompressible fows." J. Comput. Phys. 192, 262 (2003).

${ }^{42} \mathrm{M}$. Provansal, C. Mathis, and L. Boyer, "Bernard-von Karman instability: Transient and forced regimes." J. Fluid Mech. 182. 1 (1987).

${ }^{43} \mathrm{C}$. Jackson. "A finite-element study of the onset of vortex shedding in tow past variously shaped bodies," J. Fluid Mech. 182, 23 (1987).

${ }^{44}$ O. Marquet, D. Sipp. J.-M. Chonaz. and L. Jacquin. "Amplifier and resonator dynanics of a low-Reynolds number recirculation bubble in a global framework," J. Fluid Mech. 605, 429 (2008).

${ }^{45} \mathrm{P}$. Monkewitz, "The absolute and convective nature of instability in twodimensional wakes at low Reynolds numbers," Phys. Fluids 31. 999 (1988).

${ }^{46} \mathrm{X}$. Yany and Z. Zebib, "Absolute and convective instability of a cylinder wake," Phys. Fluids A 1, $689(1989)$ 